\title{
Improvement of lower urinary tract symptoms and sexual activity after open simple prostatectomy: Prospective analysis of $\mathbf{5 0}$ cases
}

\author{
Lorenzo Montesi, Luigi Quaresima, Marco Tiroli, Vito Lacetera, Ubaldo Cantoro, Giulia Sbrollini, \\ Giovanni Muzzonigro, Massimo Polito
}

Institute of Urology, AOU United Hospitals, Polytecnic University of Marche Region, Ancona, Italy.

\begin{abstract}
Summary Objectives: To evaluate the improvement of Lower Urinary Tract Symptoms

(LUTS) and Erectile Function (EF) evaluated before and after Open Simple Prostatectomy, focusing on which patients this procedure allows better outcomes in term of sexual activity.

Material and methods: 50 men with large size benign prostatic hyperplasia (BHP) greater than 80 gr were prospectively evaluated before and 6 months after Open Simple Prostatectomy (Freyer procedure) between October 2012 to September 2013. Patients had a pre-operative transrectal ultrasound (TRUS) for volume evaluation and filled pre and post operative questionnaires for International Prostate Symptom Score (IPSS) and International Index of Erectile Function (IIEF-5) score.

Results: Mean patients age was 71 years (D.S. 3,5), mean prostate volume results $103 \mathrm{ml}$ (D.S. 23,7); regarding LUTS and EF, mean improvement of IPSS score was 15,3 (D.S. 4) and mean increase of IIEF-5 score was 3,4 (D.S.3). This study highlights a correlation between patients' age and increase of IIEF-5 score; no correlation with prostate size was found.

Conclusion: According to the EAU Guidelines 2014, large size BPH (over 80-100 mL) with LUTS refractory to medical management continue to have open prostatectomy as the treatment of choice. In our experience we found not only an reduction of LUTS after the procedure but also an improvement of erectile function; this improvement was related with patient's age.
\end{abstract}

KEY WORDS: Prostate; Benign Prostatic Hyperplasia (BPH); Open simple prostatectomy; Erectile dysfunction; Lower Urinary Tract Symptoms (LUTS).

Submitted 3 October 2014; Accepted 31 October 2014

\section{INTRODUCTION}

Benign Prostatic Hyperplasia (BPH) affects the male population indiscriminalety and its incidence is increasing in relation to the raise of the population's average age. It affects approximately $5-10 \%$ of men under 40 years and up to $80 \%$ of men between $70-80$ years. The growth of the gland has been related with the level of dihydrotestosterone (DHT), although In the last years it has progressively gained credibility the hypothesis of a possible involvement of environmental factors in the pathogenesis of BPH. Inflammation seems to play a significant role, and this evidence was frequently reported in the literature. Recently Zlotta et al. (1) evaluated the association between acute and chronic inflammation and prostatic hypertrophy; from their study on cadavers was evident the association between chronic flogosis and volume of the prostate gland, with a probability to observe BPH 6.8 times greater in those with chronic inflammation than in those whitoutt. The growth of the prostate is responsible of lower urinary tract symptoms (LUTS) such as urinary frequency, nocturia, hesitancy, feeling of incomplete voiding, terminal dropping that involve a substantial reduction in quality of life (QoL) for the patient $(2,3)$.

Associated with these symptoms has evolved over time the idea that inflammatory BPH may affect the reduction of sexual potency in the old man, especially if associated with other contributing factors such as diabetes, hypertension or vascular disease. By now it is well established the beneficial role of surgery, either endoscopic or open, in the improvement of urinary symptoms. Recently, Garcia et al. (4) compared the Open Simple Prostatectomy (OSP) with laparoscopic extraperitoneal adenomectomy showing how obtain great results with this technique especially in terms of intraoperative bleeding and days of hospitalization. The technique is a feasible alternative to the intervention of OSP which is still today the gold standard procedure. More uncertain remain instead the apparent benefit on the recovery of erectile function. In 1997 Goriunov et al. (5) assessed erectile function in 818 patients undergoing to surgery for BPH. It appeared that the OSP deteriorated the erectile function (EF) of sexually active patients, but also that, in a low percentage of cases $(5 \%)$, sexual function was recovered in patients previously not sexually active. Over time, new researches have shown the association between prostatic hypertrophy and reduction of EF: with the improvement of surgical techniques, the recovery of sexual function becomes a goal to be pursued both clinically and surgically. 
Aim of our study was to evaluate the improvement of LUTS and EF valued before and after OSP, focusing in particular to show in which patients this procedure allows better outcomes in term of sexual activity.

\section{MATERIALS AND METHODS}

The study was conducted prospectively. Were included in the study patients who underwent OSP between September 2012 and February 2014. All the patients were subjected before surgery to uroflowmetry and filling of the validated questionnaires International Index of Erectile Function (IIEF5) and International Prostate Syntoms Score (IPSS). The same were repeated 6 months after the surgery, in presence of negative urine cultures and without use of phosphodiesterase-5 (PDE-5) inhibitors drugs. The surgical procedures were performed by three different surgeons with more than 10 years of experience. Exclusion criteria were drop outs at follow-up, presence of significant comorbidities and use of 5- alpha reductase inhibitor (5-ARI) and/or 5-PDE drugs in the 6 months before enrollment,

Data were analyzed with the statistical program SPSS. A value of $p<0.05$ was considered statistically significant (Figure 1).

Figure 1.

Distribution of patients' prostate sizes.

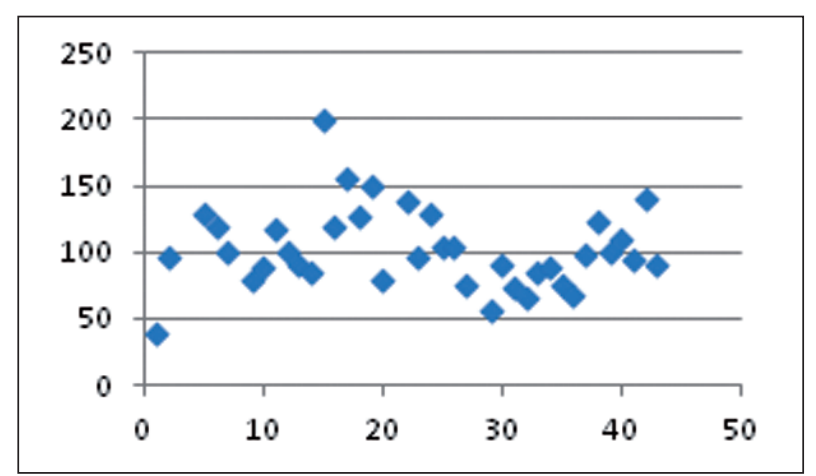

\section{RESULTS}

Fifty patients who underwent OSP between September 2012 and February 2014 were included in the study; the average age of the patients was 71 years (SD 6.5), mean prostate volume was $103 \mathrm{ml}$ (SD 33.7) with a maximum volume of $200 \mathrm{ml}$ and a minimum of $40 \mathrm{ml}$. Six patients showed preoperatively at least one episode of acute retention of urine and 4 of them came to surgery with catheter placed for chronic retention. Forty-one patients were treated at least once with alpha-blockers, while 22 of them have been submitted at least once to therapy with 5-ARI but not in the months before completing the questionnaire. The average improvement in IPSS was 15.3 (DS 8) with a maximum value of 32 and a minimum value of 0 (Figure 2).

The average improvement of IIEF5 was 3.4 (DS 5) with maximum improvement of 19 and minimum improvement of 0 Mean improvement in subgroup of patients less than 70 years resulted 4.6 (DS 5,3).
Figure 2.

IPSS scores pre and 6 months after OSP procedure.

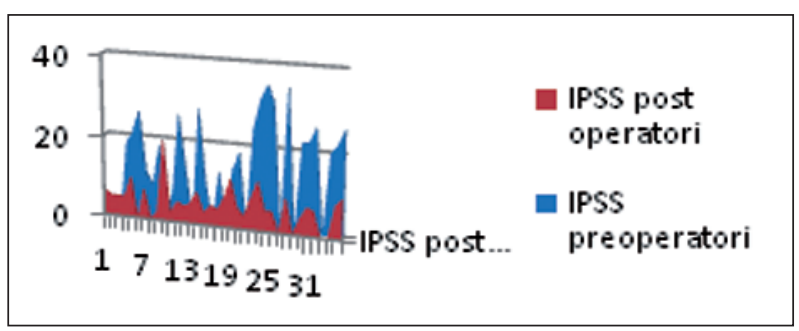

Figure 3.

IIEF-5 scores pre and 6 months after OSP procedure

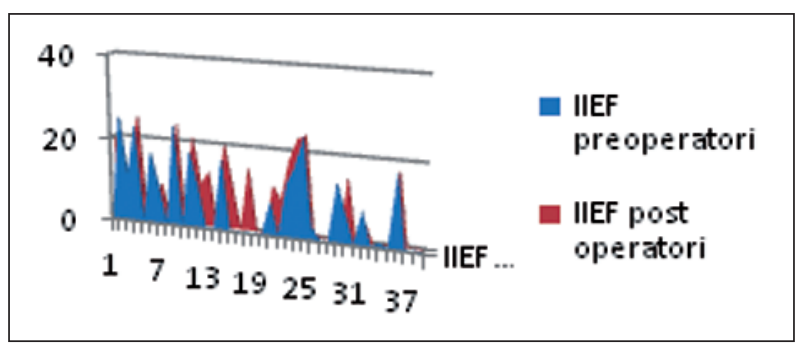

No difference between patients with different prostate size was observed.

Six patients had positive urine cultures at subsequent checks for which it was set an appropriate antibiotic therapy; one patient had wound infection.

No episode of acute retention occurred in our study in the six months follow-up after surgery and in none of the patients it was necessary to reset the alpha-blocking therapy (Figure 3).

\section{Discussion}

Our study proved to be concurring with the current European guidelines for the treatment of prostatic hypertrophy.

In fact, in agreement with the EAU Guidelines 2014, $\mathrm{BPH}$ with high volume (greater than $80 \mathrm{ml}$ ) had as first line treatment the open simple prostatectomy procedure (6-8). New techniques have been compared with OSP: Raimbault et al. (9) have compared the results obtained with photo-selective vaporization of the prostate to those obtained by OSP in high volume prostates $(>80 \mathrm{ml})$ : it resulted a lower cost compared to the benefit obtained and an inferior percentage of reoperations in the one year follow-up. Kim et al. (10) have recently tested the effect of the holmium laser enucleation of the prostate demonstrating a good improvement of the sexual function of patients, especially when associated with an improvement of irritative LUTS. To sum up, a huge number of studies in international literature have compared surgical results of different techniques, some of them analyzed the EF, but few studies tried to find prognostic factors to predict which category of patients has the best outcome in term of improvement of EF after OPS; in our experience the only patients characteristic that predict EF outcome was patients age. 


\section{Conclusions}

The results obtained show how OSP procedure provides excellent results as regards the obstructive voiding symptoms. Encouraging results were also obtained with regard to the recovery of sexual potency, in particular correlated to the patient's age; additional studies with a bigger sample size are strongly recommended to confirm this theory.

\section{References}

1. Zlotta AR, Egawa S, Pushkar D, et al. Prevalence of inflammation and benign prostatic hyperplasia on autopsy in asian and caucasian men. Eur Urol. 2014; 66:619-22.

2. Abrams P, Cardozo L, Fall M, et al. The standardisation of terminology of lower urinary tract function:report from the Standardisation Sub-committee of the International Continence Society. Neurourol Urodyn. 2002; 21:167-78.

3. Chapple CR, Wein AJ, Abrams P, et al. Lower urinary tract symptoms revisited: a broader clinical perspective. Eur Urol. 2008; 54:563-9.

4. García-Segui A, Gascón-Mir M. Comparative study between laparoscopic extraperitoneal and open adenomectomy. Actas Urol Esp. 2012; 36:110-6.

5. Goriunov VG, Davidov MI. Sexual readaptation after the surgical treatment of benign prostatic hyperplasia. Urol Nefrol (Mosk). 1997; (5):20-4

6. Tubaro A, Carter S, Hind A, et al. A prospective study of the safety and efficacy of suprapubic transvesical prostatectomy in patients with benign prostatic hyperplasia. J Urol. 2001; 166:172-6.

7. Mearini E, Marzi M, Mearini L, et al. Open prostatectomy in benign prostatic hyperplasia: 10-yearexperience in Italy. Eur Urol 1998; 34:480-5.

8. Serretta V, Morgia G, Fondacaro L, et al. Open prostatectomy for benign prostatic enlargement in southern Europe in the late 1990s: a contemporary series of 1800 interventions. Urology. 2002; 60:623-7.

9. Raimbault M, Watt S, Bourgoin H, et al. Comparative analysis of photoselective vaporization of the prostate with the Greenlight laser and open prostatectomy for high volume prostate hypertrophy. Prog Urol. 2014; 24:470-6.

10. Kim SH, Yang HK, Lee HE, Paick JS, Oh SJ. HoLEP does not affect the overall sexual function of $B P H$ patients: a prospective study. Asian J Androl. 2014.

\section{Correspondence}

Lorenzo Montesi, MD

lorenzomontesi@yahoo.it

Luigi Quaresima, MD (Corresponding Author)

luigiquaresima@yahoo.it

Marco Tiroli, MD

marcotiroli@libero.it

Vito Lacetera, MD

vlacetera@gmail.com

Ubaldo Cantoro, MD

ubymaior@libero.it

Giulia Sbrollini, MD

giuliasbrollini@libero.it

Giovanni Muzzonigro, MD

g.muzzonigro@univpm.it

Massimo Polito, MD

max_polito@virgilio.it

Institute of Urology, AOU United Hospitals,

Polytecnic University of Marche Region, Ancona, Italy 